\title{
Modeling Propagation of Shock Waves in Metals
}

W. M. Howard, J. D. Molitoris

August 30, 2005

APS Topical SCCM Meeting

Baltimore, MD, United States

July 31, 2005 through August 5, 2005 
This document was prepared as an account of work sponsored by an agency of the United States Government. Neither the United States Government nor the University of California nor any of their employees, makes any warranty, express or implied, or assumes any legal liability or responsibility for the accuracy, completeness, or usefulness of any information, apparatus, product, or process disclosed, or represents that its use would not infringe privately owned rights. Reference herein to any specific commercial product, process, or service by trade name, trademark, manufacturer, or otherwise, does not necessarily constitute or imply its endorsement, recommendation, or favoring by the United States Government or the University of California. The views and opinions of authors expressed herein do not necessarily state or reflect those of the United States Government or the University of California, and shall not be used for advertising or product endorsement purposes.

This work was performed under the auspices of the U.S. Department of Energy by University of California, Lawrence Livermore National Laboratory under Contract W-7405-Eng-48. 


\title{
MODELING PROPAGATION OF SHOCK WAVES IN METALS
}

\author{
W. M. Howard, and J. D. Molitoris \\ University of California, Lawrence Livermore National Laboratory, Livermore CA 94551
}

\begin{abstract}
We present modeling results for the propagation of strong shock waves in metals. In particular, we use an arbitrary Lagrange Eulerian (ALE3D) code to model the propagation of strong pressure waves ( $\mathrm{P} \sim 300$ to 400 kbars) generated with high explosives in contact with aluminum cylinders. The aluminum cylinders are assumed to be both flat-topped and have large-amplitude curved surfaces. We use 3D Lagrange mechanics. For the aluminum we use a rate-independent Steinberg-Guinan model, where the yield strength and shear modulus depend on pressure, density and temperature. The calculation of the melt temperature is based on the Lindermann law. At melt the yield strength and shear modulus is set to zero. The pressure is represented as a seven-term polynomial as a function of density. For the HMX-based high explosive, we use a JWL, with a program burn model that give the correct detonation velocity and C-J pressure ( $\mathrm{P} \sim 390 \mathrm{kbars}$ ). For the case of the large-amplitude curved surface, we discuss the evolving shock structure in terms of the early shock propagation experiments by Sakharov,.
\end{abstract}

\section{INTRODUCTION}

We model the propagation of strong shock waves in aluminum and compare our results with experimental radiographic images. The shock waves are generated with an HMX-based explosive that is placed in direct contact with a flat-topped cylinder, which in turn, is in direct contact with an aluminum ejecta plate. Radiographic images are taken at various times as the shock wave transits the aluminum cylinder and emerge from the ejecta plate $^{1}$. From these images the shock structures can be determined. These images are compared to model calculations of the detonation wave in the high explosive and the propagation of this shock into the aluminum cylinder and ejecta plate. In future experiments and models, we plan to study shock waves onto cylinders with large-amplitude curved-surfaces. Our goal is to relate theses results to the original Sakharov experiments ${ }^{2}$, in order to infer the viscosity of the aluminum from the damping rate of the curved shock fronts.

\section{HYDRODYNAMICS AND MATERIAL STRENGTH MODELS}

We use a 3-D Arbitrary-Lagrange-Eulerian (ALE3D) code $^{3}$ with implicit or explicit coupled thermal transport, advection and a variety of material models for metals and high explosives. These models may include strength, fracture, melting and spall. For this calculation, we have used compressible flow with explicit Lagrange mechanics. We use a 3-D mesh with fine zoning (40 zones $/ \mathrm{cm}$ ) in the direction of propagation of the shock wave and a butterfly mesh in the transverse 
direction. Figure 1 shows the experimental and model configuration and Figure 2 shows an example of the mesh.

Figure 2. Numerical grids showing the aluminum cylinder and the expanding gases from the detonation of the high explosive. The figure on the bottom is a view of the grids from the top, showing the butterfly mesh. This zoning allows a 3-D rendering of the experiment.

\section{EXPERIMENTAL RESULTS}

Figure 3 shows a sequence of radiographic images from the time that the shock wave enters the aluminum to the time that it emerges from the ejecta plate and beyond ${ }^{1}$. The experimental structure of the aluminum and the timing of the propagation of the shock wave are replicated in the model calculations. At late times, after the emergence of the shock wave from the ejecta plate, the aluminum cylinder becomes highly distorted.

For the high explosive we use a JWL, with a program burn model that gives the correct detonation velocity $(7.27 \mathrm{~km} / \mathrm{s})$ and C-J pressure ( $\sim 390$ kbars). The detonation wave is represented as a plane wave in the high explosive. For the aluminum we use a Steinberg-Guinan isotropic strength model $^{4}$, where the yield strength and shear modulus depend on the pressure and temperature. The calculation of the melt temperature is based on the Lindermann law. Above melt temperature the yield strength and shear modulus are set to zero. The pressure is represented as a seven-term polynomial as a function of density. The initial shear modulus is taken to be $0.276 \mathrm{Mbar}$ and the initial yield strength is taken to be 2.9 kbars. We use a constant heat capacity and thermal conductivity for the aluminum. These initial calculations do not include a spall model. For the Lucite, we use a Mie-Gruneisen equation of state with a constant yield strength and shear modulus.

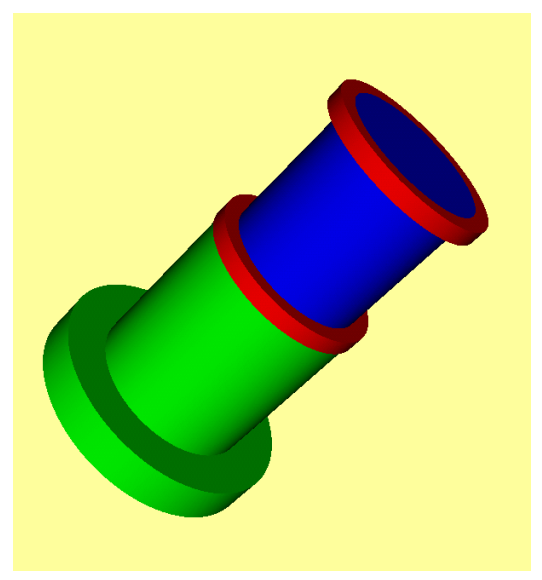

Figure 1. Experimental and modeling configuration. The Lucite rings are in red, the HMX-based explosive is in blue and the aluminum cylinder and ejecta plate are in green.
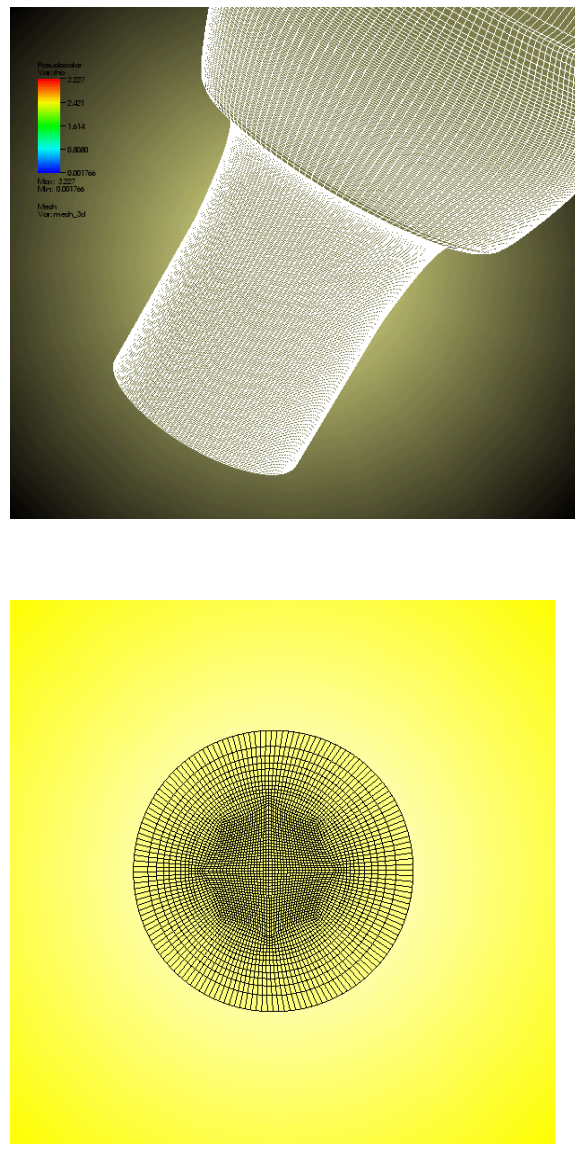

Figure 2. Numerical grids showing the aluminum cylinder and the expanding gases from the detonation of the high explosive. The figure on the bottom is a view of the grids from the top, showing the butterfly mesh. This zoning allows a 3-D rendering of the experiment.

\section{EXPERIMENTAL RESULTS}

Figure 3 shows a sequence of radiographic images from the time that the shock wave enters the 
aluminum to the time that it emerges from the ejecta plate and beyond ${ }^{1}$. The experimental structure of the aluminum and the timing of the propagation of the shock wave are replicated in the model calculations. At late times, after the emergence of the shock wave from the ejecta plate, the aluminum cylinder becomes highly distorted.

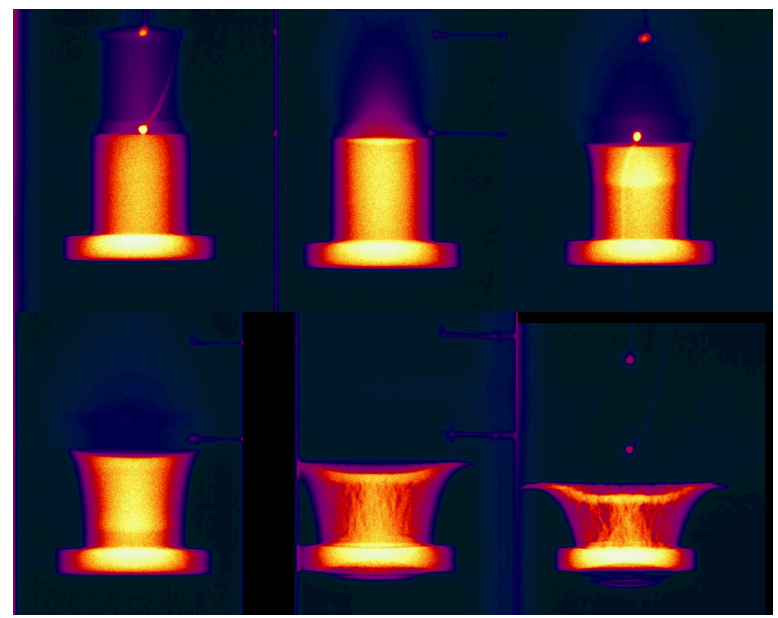

Figure 3. Experimental radiographic images of aluminum cylinder and spall plate. One can observe the shock wave as it transverses the cylinder and emerges from the spall plate
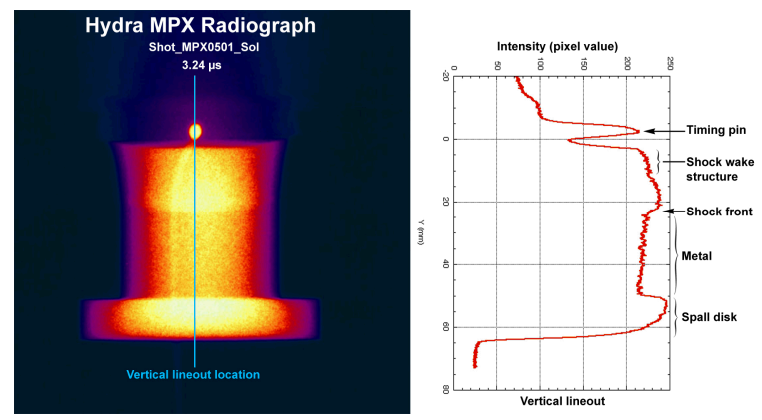

Figure 4. Radiographic intensity revealing the shock structure in the aluminum cylinder.

\section{MODEL RESULTS}

Figure 5 shows the calculated density structure in the aluminum cylinder at approximately the same time as the experimental image shown in Figure 4. The overall shape of the cylinder and shock front agree with the experiment. However, the lineout reveals that the density structure due to the shock wave is rather sharp, with a rare faction wave behind the shock front. At the time that the shock wave approaches the eject plate, the pressure is approximately $100 \mathrm{kbars}$ and approximately 30 kbars it emerges from the ejecta plate. Figure 6 shows the ejecta velocity, as the shock wave emerges from the ejecta plate. The value of this velocity agrees with the experimental measurement.
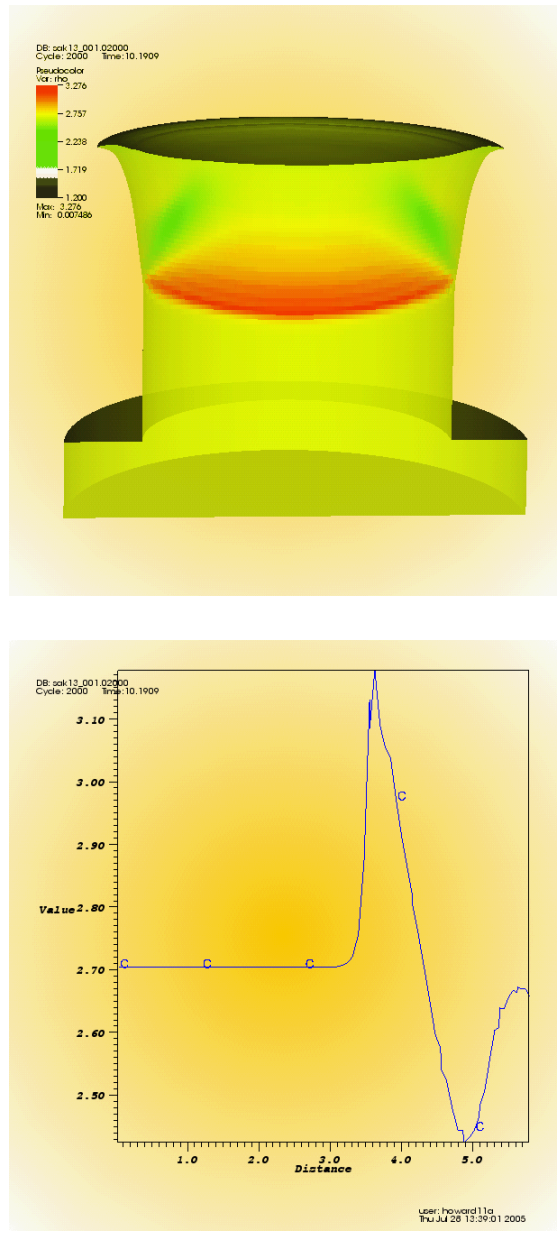

Figure 5. Calculation of the density structure of the aluminum at approximately the same time as Figure 4. The lineout reveals a rather sharp density structure due to the shock. The green 
areas on the left are lower density structures where the aluminum is in tension.

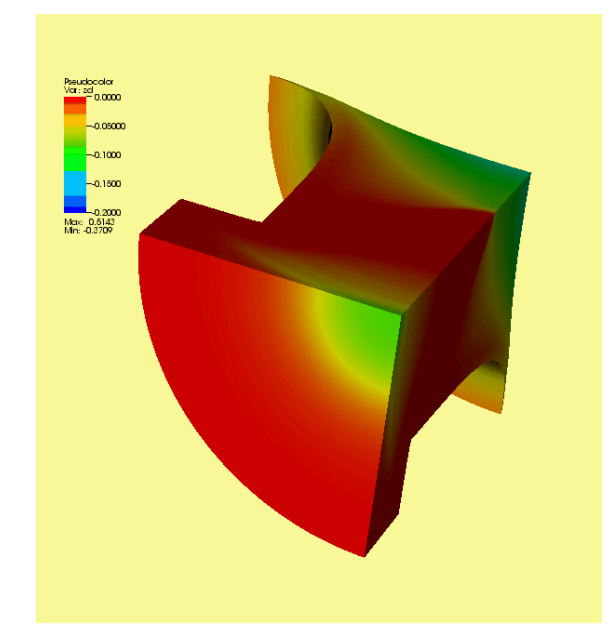

Figure 6. The spall ejection velocity (shown in green) of $\sim 0.7 \mathrm{~mm} / \mu \mathrm{s}$ at the time of the emergence of the shock front from the ejecta disk. Experiments at HEAF using the Hydra multi-channel $x$-ray imaging system measure the spall velocity of the ejecta disk to be 0.66 $\mathrm{mm} / \mu \mathrm{s}$. This measurement was consistent over two independent data sets.

\section{CONCLUSIONS}

We have modeled a series of experiments on taking radiographic images of the propagation of strong shock waves in aluminum. We have demonstrated that our hydrodynamics and material models can successfully replicate the experimental results for the case of planer shocks. We predict the shock structure, propagation times and spall velocities. In the future we plan to apply these models to study the propagation of strongly curved shocks and relate the results to the original Sakharov experiments.

\section{ACKNOWLEDGEMENTS}

We would like to thank the ALE3D development team, particularly A. Nichols and B. Wallin for helpful assistance

\section{REFERENCES}

1. Molitoris, J. D., R. G. Garza, W. M. Howard, H. G. Andreski and L. D. Couch, "High-Explosive Shock Compression of Aluminum through Smooth and Corrugated Surfaces", these proceedings.

2. Sakharov, A. D., Dokl Akad Nauk SSSR, 159, 1019, 1964.

3. Nichols III, A. L., A. Anderson, R. Neely and B. Wallin, $12^{\text {th }}$ International Detonation Symposium, San, Diego, Office of Naval Research, 2002.

4. Steinberg, D. J., S. G.Cochran and M. W. Gruinan, J. Applied Physics, 51, 1498, 1980. 